\title{
Enhanced Characterization of Singly Protonated Phosphopeptide Ions by Femtosecond Laser-induced Ionization/Dissociation Tandem Mass Spectrometry (fs-LID-MS/MS)
}

\author{
Scott A. Smith, ${ }^{\text {a }}$ Christine L. Kalcic, ${ }^{\text {a }}$ Kyle A. Safran, ${ }^{\text {a }}$ Paul M. Stemmer, ${ }^{\text {b }}$ \\ Marcos Dantus, ${ }^{\mathrm{a}, \mathrm{c}}$ and Gavin E. Reid ${ }^{\mathrm{a}, \mathrm{d}}$ \\ ${ }^{a}$ Department of Chemistry, Michigan State University, East Lansing, Michigan, USA \\ ${ }^{\mathrm{b}}$ Institute of Environmental Health Sciences, Wayne State University, Detroit, Michigan, USA \\ ${ }^{c}$ Department of Physics, Michigan State University, East Lansing, Michigan, USA \\ d Department of Biochemistry and Molecular Biology, Michigan State University, East Lansing, Michigan, \\ USA
}

To develop an improved understanding of the regulatory role that post-translational modifications (PTMs) involving phosphorylation play in the maintenance of normal cellular function, tandem mass spectrometry (MS/MS) strategies coupled with ion activation techniques such as collision-induced dissociation (CID) and electron-transfer dissociation (ETD) are typically employed to identify the presence and site-specific locations of the phosphate moieties within a given phosphoprotein of interest. However, the ability of these techniques to obtain sufficient structural information for unambiguous phosphopeptide identification and characterization is highly dependent on the ion activation method employed and the properties of the precursor ion that is subjected to dissociation. Herein, we describe the application of a recently developed alternative ion activation technique for phosphopeptide analysis, termed femtosecond laser-induced ionization/dissociation (fs-LID). In contrast to CID and ETD, fs-LID is shown to be particularly suited to the analysis of singly protonated phosphopeptide ions, yielding a wide range of product ions including $a, b, c, x, y$, and $z$ sequence ions, as well as ions that are potentially diagnostic of the positions of phosphorylation (e.g., ' $a_{n}+1-98$ '). Importantly, the lack of phosphate moiety losses or phosphate group 'scrambling' provides unambiguous information for sequence identification and phosphorylation site characterization. Therefore, fs-LID-MS/MS can serve as a complementary technique to established methodologies for phosphoproteomic analysis. (J Am Soc Mass Spectrom 2010, 21, 2031-2040) (c) 2010 Published by Elsevier Inc. on behalf of American Society for Mass Spectrometry

$\mathrm{O}$ $f$ the multitude of protein post-translational modifications (PTMs) that occur in vivo to regulate normal cellular function, and whose dysregulation is associated with the onset and progression of disease, phosphorylation is perhaps the most prevalent [1-4]. Hence, significant efforts have been extended in recent years toward the development of robust analytical methodologies for 'phosphoproteome' analysis [5-7]. In a typical phosphoproteomic analysis strategy, proteolytically derived phosphopeptides are first enriched [8], then subjected to tandem mass spectrometry (MS/MS) analysis to determine the identity (i.e., the sequence) of the peptide, as well as to localize the site of the phosphate modification to a specific residue within the peptide sequence $[9,10]$. Numerous ion activation techniques have been employed in con-

Address reprint requests to Dr. G. E. Reid, Department of Chemistry, Michigan State University, 229 Chemistry Building, East Lansing, MI, 48824, USA. E-mail: reid@chemistry.msu.edu junction with MS/MS for phosphopeptide analysis [7]. The most commonly used ion activation methods to date are low-energy collision-induced dissociation (CID) and electron-transfer dissociation (ETD). Characteristics of CID typically include high-efficiency fragmentation, simple instrumentation requirements, and the production of a relatively simple series of $b$ - and $y$-type 'sequence' ions resulting from fragmentation of protonated amide functionalities along the peptide backbone. One of the limitations of CID, however, is the dominant 'non-sequence' loss of the phosphate moiety [i.e., phosphoric acid $\left(\mathrm{H}_{3} \mathrm{PO}_{4} ; 98 \mathrm{Da}\right)$ or metaphosphate $\left.\left(\mathrm{HPO}_{3} ; 80 \mathrm{Da}\right)\right]$, particularly from precursor ions under 'non-mobile' and 'partially-mobile' proton mobility (i.e., typically low charge state) conditions [11, 12]. The observation of these non-sequence losses as dominant product ion fragmentation pathways can reduce the abundance of sequence ions, and may therefore hinder subsequent characterization of the phosphopeptide sequence and the site(s) of phosphorylation. Although 
loss of the phosphate group during ion activation is undesirable with respect to unambiguous sequence and phosphorylation site assignment, it should be noted that such losses can be useful as a diagnostic indication of which species in an unknown sample are phosphorylated. Indeed, data-dependent MS/MS strategies have been developed in which the observation of CIDinduced phosphate losses are used to trigger subsequent activation of the same species with another technique [e.g., ETD or electron capture dissociation (ECD)] to facilitate unambiguous sequence identification (provided the precursor ion charge state is compatible with the second technique) [13]. In addition to the potential for phosphate group losses, phosphopeptide ions subjected to CID in ion trap mass spectrometers have also been observed to exhibit phosphate group 'scrambling' before dissociation, resulting in the formation of product ions that may lead to erroneous assignment of the site of phosphorylation within the peptide [12]. Similar to fragmentation reactions involving loss of the phosphate group, this undesirable effect is most pronounced under non-mobile and partially-mobile protonation conditions. Hence, CID-MS/MS methods should only be considered to be reliable for unambiguous phosphorylation site assignment when product ions corresponding to these losses or rearrangement reactions are not observed as abundant species. We note that two reports have recently been published indicating that product ions resulting from gas-phase rearrangement reactions are not observed as abundant species using conventional automated ESI-CID-MS/MS analysis strategies (where the precursor ions selected for dissociation are typically under mobile or partially-mobile protonation conditions), and therefore do not have an appreciable effect on the reliability of PTM site localization in phosphoproteomic datasets [14, 15].

Under circumstances where extensive neutral losses or rearrangement reactions are observed, electron capture dissociation (ECD) [16] or electron-transfer dissociation (ETD) [17-19] may potentially be employed to overcome the limitations of CID. (Note that ECD and ETD differ primarily by the mechanism of electron delivery to the analyte, with subsequent fragmentation being near-identical; as such, the discussion in the remainder of this text will refer only to ETD for the sake of brevity.) During ETD, electron-transfer between a singly-charged reagent anion and a multiply-charged precursor cation results in the formation of an unstable reduced odd-electron intermediate which undergoes radical-initiated decomposition to yield primarily $c$ and $z$-type sequence ions. This dissociation is relatively nonselective with respect to the residues at which dissociation occurs [20], allowing a complete or nearcomplete set of sequence ions of similar abundance to be formed, while losses of the phosphate moiety or phosphate group scrambling are not observed. Thus, ETD-MS/MS methods can be considered to be generally reliable for unambiguous phosphorylation site assignment. One limitation to ETD, however, is that it is not compatible with the dissociation of singly charged cations [for example, those formed by using matrix assisted laser desorption ionization (MALDI)]. Further, ETD is most effectively performed on precursor ions with relatively high charge states (i.e., $\geq 3+$ ) where the ion/ion reaction cross-section is sufficiently large for efficient dissociation to occur [21]. Thus, it is often the case that doubly-protonated species do not efficiently undergo a dissociative reaction in a reasonably short time period to facilitate rapid analysis. It is also known that some post-ETD product pairs may not efficiently fragment due to strong intermolecular cohesion of the fission products. For example, strong noncovalent gasphase interactions have been observed between the phosphate group of phosphorylated serine, threonine, and tyrosine residues and the side chains of lysine and arginine residues [22]. To remedy this, supplemental collisional activation may be applied to disrupt such bonds [23]. However, the addition of collisional heating could potentially contribute to phosphate loss or/and rearrangements, resulting in ambiguity to the phosphorylation site assignment.

Note that the conditions that lead to the most effective ETD fragmentation (i.e., high charge state precursors) are also those where CID is most effective (i.e., where losses of the phosphate moiety or rearrangement reactions are minimized). Thus, it can be argued that there is significant overlap in the applicability of these two techniques for phosphopeptide characterization. As a consequence, there remains a need for the development of alternate ion activation strategies for phosphopeptide analysis, particularly for the effective dissociation of low charge state precursor ions.

Photodissociation strategies are increasingly being evaluated as alternatives to CID and ETD for peptide and phosphopeptide characterization. With 'slow heating' techniques such as infrared multiphoton dissociation (IRMPD), the trapped precursor ion population is irradiated with low-energy photons $(0.1 \mathrm{eV} /$ photon for a typical $10.6 \mu \mathrm{m} \mathrm{CO}$ laser) for a period ranging from a few milliseconds to several seconds to effect amide bond fragmentation and the formation of $b$ - and $y$-type sequence ions [24]. From an analytical perspective, IRMPD of phosphopeptide ions produces product ion spectra that are generally comparable to low-energy CID, and could therefore suffer from similar limitations to those outlined above. In contrast, photodissociation in the ultraviolet (i.e., UVPD) wavelength range (157$355 \mathrm{~nm} ; 7.9-3.5 \mathrm{eV})$, by which prompt dissociation occurring from excited state species typically yields $a$ or $x$-type product ions from singly-protonated peptides, as well as $d-, v$, and $w$-ions resulting from side-chain cleavages, depending on the location of the charge in the peptide and the observation time frame for the experiment $[25,26]$. Interestingly, it has been recently reported that $193 \mathrm{~nm}$ UVPD in a tandem time-of-flight (TOF-TOF) instrument yields characteristic $a_{n}+1-98$ product ions when the $n^{\text {th }}$ residue is occupied by a 
phosphorylated species, suggesting that UVPD-MS/MS may offer unique diagnostic information for phosphorylation site assignment [27]. However, a recent study involving time resolved $157 \mathrm{~nm}$ UVPD of singly charged arginine-terminated phosphopeptides in an ion trap-TOF mass spectrometer revealed that abundant neutral losses of the phosphate group from the precursor ions are observed just $300 \mathrm{~ns}$ after photoactivation, and that phosphate group losses from the product ions became common after just $13 \mu$ s [28].

Recently, an alternative photodissociation technique termed 'femtosecond laser-induced ionization/dissociation' (fs-LID) has been introduced [29]. Despite the relatively low energies of the near-infrared photons ( $\sim 800 \mathrm{~nm} ; 1.55 \mathrm{eV} /$ photon) that are employed using this technique, a coherent packet of $\sim 1 \times 10^{15}$ photons made to pass by an ion on an ultra-short timescale $(\sim 35$ fs, FWHM) is of such intensity (peak power $4.6 \times 10^{14}$ $\mathrm{W} / \mathrm{cm}^{2}$ ) that its electric field is capable of warping the ion's pseudo-potential energy surface to a sufficient extent so as to allow an electron to escape via a tunneling ionization mechanism, on a timescale similar to or faster than that of bond vibrations (tens to hundreds of fs) [30]. The resultant oxidized radical intermediate species (e.g., $[\mathrm{M}+\mathrm{H}]^{+} \rightarrow[\mathrm{M}+\mathrm{H}]^{2+}$ ) can then undergo relatively nonselective dissociation to produce a wide variety of peptide sequence ions $(a, b, c, x, y$, and $z$ ). Thus, similar to ETD, fs-LID is characterized by radical-directed dissociation pathways where losses or rearrangements of potentially labile functionalities such as the phosphate group are minimized [29]. Another notable feature of fs-LID is that precursor ions of low charge state are observed to undergo the greatest fragmentation efficiency, a phenomenon attributed to their lower ionization potential compared with higher charge states [31]. Similar dependences on ionization potential have been previously reported for electron ionization dissociation (EID) in FT-ICR mass spectrometers [31] as well as for Penning ionization following interaction with metastable atoms [metastable atom dissociation, (MAD)] in ion trap mass spectrometers [32, 33]. These results suggest that fs-LID-MS/MS could serve as a complementary methodology for characterization of the phosphopeptide precursor ions, which are currently least reliably analyzed by CID-MS/MS, or are not amenable to ETD-MS/MS, i.e., singly protonated ions. One of the limitations of our original report on fs-LID MS/MS, however, was relatively poor dissociation efficiency, regardless of the precursor ion charge state, which can be attributed in part to the performance of the laser hardware used [29]. The laser system used in that publication was capable of $300 \mu \mathrm{J} /$ pulse at a $1 \mathrm{kHz}$ repetition rate. Here, we demonstrate that replacement of the laser system with one that delivers $330 \mu \mathrm{J} /$ pulse at a $10 \mathrm{kHz}$ repetition rate results in approximately one order of magnitude improvement in fragmentation efficiency for a comparable irradiation period.

\section{Materials and Methods}

\section{Sample Preparation}

The phosphopeptides used in this study were synthesized either in-house or by Sigma-Aldrich (St. Louis, MO, USA), and had the following sequences: LFPTGHPESLER, HEVSASpTQpSTPASSR, LNQSpSPDNVTDTK, HYGIpTSPISLAAPK, EDPSGTFSLGK, and SLSSPPTDNLELSLR, where the lower-case ' $\mathrm{p}$ ' indicates that the following residue is phosphorylated. Water (18.2 M $\mathrm{M}$ $\mathrm{cm})$ was obtained from a Barnstead Nanopure Diamond filtration system (Dubuque, IA, USA). Methanol $(\mathrm{MeOH})$ was purchased from J. T. Baker (Phillipsburg, NJ, USA). Acetic acid $\left(\mathrm{CH}_{3} \mathrm{CO}_{2} \mathrm{H}\right)$ was from Mallinckrodt (Phillipsburg, NJ, USA). Sample solutions (2-20 $\mu \mathrm{M})$ were prepared in $\mathrm{MeOH} / \mathrm{H}_{2} \mathrm{O} / \mathrm{CH}_{3} \mathrm{CO}_{2} \mathrm{H}$ (49.5: 49.5:1.0\% volume).

\section{$f_{S}$-LID-MS/MS and CID-MS/MS}

A modified 3D quadrupole ion trap mass spectrometer (LCQ Deca XP Plus; Thermo Scientific, San Jose, CA) was used for fs-LID-MS/MS and CID-MS/MS analyses. A version of this instrumentation has been previously described [29]. However, in the present study, a new laser system was installed to provide both greater power and higher laser pulse repetition rate. To create laser pulses with durations on the femtosecond timescale, a broadband Ti: $\mathrm{Al}_{2} \mathrm{O}_{3}$ oscillator (Ti-Light model; Quantronix, East Setauket, NY, USA) was used to seed a two-stage amplified Ti: $\mathrm{Al}_{2} \mathrm{O}_{3}$ laser (Integra-HE model, Quantronix), which was phase-shaped using a MIIPS Box Pulse Shaper (Biophotonics Solutions, Okemos, MI, USA) to yield transform-limited pulses [34]. The near-IR output from the amplifier (798 $\pm 14 \mathrm{~nm}$ (FWHM)) was $\sim 35 \mathrm{fs}$ (FWHM) in duration, with a repetition rate of $10 \mathrm{kHz}$. The laser pulses $(330 \mu \mathrm{J} /$ pulse) were focused into the mass spectrometer using a periscope and a lens of $400 \mathrm{~mm}$ focal length, yielding a calculated peak power of $4.6 \times 10^{14} \mathrm{~W} / \mathrm{cm}^{2}$. The laser beam was passed into the vacuum manifold via a fused silica view port, beyond which the laser beam was directed through the ring electrode of the mass analyzer via a hole centered on and co-planar to the $\mathrm{z}=0$ plane of the ion trap. To prevent interfering signal due to scattered light causing ablation of or/and photoelectron emission from the trap electrodes, the beam was passed through the far side of the ring electrode (through another $5 \mathrm{~mm}$ hole) and out of the manifold through a view port. To perform fs-LID during the ion activation period of the mass analyzer scan function, a beam shutter was triggered automatically via a TTL output signal from TP_15 of the mass spectrometer, controlled from the advanced Diagnostics menu within the Tune Plus window of the Xcalibur software.

Peptide ions were introduced to the mass spectrometer using an Advion Nanomate Triversa (Advion BioSciences, Ithaca, NY, USA) nano-electrospray ioniza- 
tion (nESI) source operating in infusion mode. Chipsoft ver. 7.1 software (Advion, Ithaca, NY, USA) was used to set the NanoMate spray voltage to $1.5 \mathrm{kV}$, gas pressure to $0.3 \mathrm{psi}$, and post-aspiration air gap of $3 \mu \mathrm{L}$. The ion transfer tube of the mass spectrometer was maintained at $200{ }^{\circ} \mathrm{C}$.

Precursor ions were isolated and subjected to positive ion mode CID-MS/MS and fs-LID-MS/MS. Selected product ions formed by fs-LID were subjected to CID-MS $^{3}$. To prevent contamination of the fs-LIDMS/MS data by CID product ions that may arise from off-resonant excitation during isolation, precursor ions were 'double isolated' by using a first stage of isolation with a narrow isolation window $(1.5-3 \mathrm{~m} / \mathrm{z})$ followed by a second isolation stage using a relatively broad isolation window $(4-10 \mathrm{~m} / \mathrm{z})$, with the window breadth being dependent on ion $\mathrm{m} / \mathrm{z}$ and charge state. Fs-LID activation periods of $100 \mathrm{~ms}$, corresponding to total laser exposure times of a few tens of picoseconds, were sufficient to typically dissociate $>50 \%$ of the precursor population (determined by measuring the ratio of the post-acquisition precursor ion abundance:preacquisition precursor ion abundance). The focused beam diameter was calculated to be approximately $60 \mu \mathrm{m}$. Depending on the $\mathrm{m} / \mathrm{z}$ of the precursor ion and the applied rf, the ion cloud is in the order of several hundreds of $\mu \mathrm{m}$ in diameter. Although the exact position of the laser with respect to the ion cloud was not known, the position of the laser was manually tuned to achieve 'optimal' dissociation efficiency. For CID, the default activation period of $30 \mathrm{~ms}$ was used. All spectra were acquired with the same Mathieu q-values $\left(\mathrm{q}_{\mathrm{z}}=\right.$ 0.25 ) and Automatic Gain Control settings (MS/MS AGC target $=2.00 \mathrm{E} 7$ ).

\section{ETD-MS/MS}

ETD-MS/MS data were acquired using standard conditions on a commercial linear quadrupole ion trap (Thermo Scientific LTQ XL) equipped with a negative chemical ionization source for generation of the ETD reagent anions (fluoranthene).

\section{Data Analysis}

To allow a detailed comparison of the structural information that could be obtained by interrogation of the product ions contained within the respective fs-LIDand CID-MS/MS spectra, all MS/MS spectra were acquired for a period of 2-5 $\mathrm{min}$, then averaged. The extent of signal averaging depended on parameters such as the initial precursor ion abundance, precursor ion dissociation efficiency, and the number of product ions formed. Typically, $\sim 10 \mu \mathrm{M}$ solutions were used. Thus, assuming a $200 \mathrm{~nL} / \mathrm{min}$ flow rate and a peptide of $1000 \mathrm{Da}, 1 \mu \mathrm{L}(10 \mathrm{pmol})$ of sample would be consumed for a $5 \mathrm{~min}$ acquisition. However, for a $10 \mathrm{~s}$ acquisition period (see the Results and Discussion section below), only $330 \mathrm{fmol}$ peptide would be consumed.
All spectra were subjected to a five-point Gaussian smooth before analysis. Fs-LIDMS/MS spectra were compared with isolation and background data (i.e., an identical MS/MS experiment performed with solvent only) to avoid erroneous assignment of product ions formed by background fs-LID. Manual peak picking was facilitated with the MS Product program (Protein Prospector, ver. 5.5.0; http://prospector.ucsf.edu). In the figures, the following chemical groups are represented symbolically for clarity: $\Delta=$ loss of phosphoric acid $\left(\mathrm{H}_{3} \mathrm{PO}_{4}, 98 \mathrm{Da}\right)$ or the combined losses of metaphosphate and water $\left(\mathrm{HPO}_{3}+\mathrm{H}_{2} \mathrm{O}, 98 \mathrm{Da}\right) ; \square=$ loss of metaphosphate $\left(\mathrm{HPO}_{3}, 80 \mathrm{Da}\right) ; \mathbf{\square}=$ addition of metaphosphate $\left(\mathrm{HPO}_{3}, 80 \mathrm{Da}\right) ; *=$ loss of ammonia $\left(\mathrm{NH}_{3}, 17\right.$ Da); $\mathrm{O}=$ loss of water $\left(\mathrm{H}_{2} \mathrm{O}, 18 \mathrm{Da}\right) ; \Psi=$ loss of $\mathrm{CH}_{2} \mathrm{O}$ $(30 \mathrm{Da}) ; \#=$ loss of $\mathrm{CO}_{2}$ or $\mathrm{HNCHNH}_{2}(44 \mathrm{Da}) ; \pi=$ loss of $58 \mathrm{Da} ; \xi=$ loss of $56 \mathrm{Da}$.

\section{Results and Discussion}

To demonstrate the complementarity of fs-LID to CID and ETD for phosphopeptide characterization as well as to evaluate the types of ions formed, the prevalence of phosphate group losses or/and rearrangement and the fragmentation efficiency as a function of charge state, a series of phosphopeptides have been analyzed by fsLID-, CID-, and ETD-MS/MS. Shown in Figure 1a is the product ion spectrum obtained by fs-LID-MS/MS of the singly protonated precursor ion from the singly phosphorylated peptide LFpTGHPESLER. The abundance of the isolated precursor ion before laser irradiation was 1.88E7 (arbitrary units, data not shown) while the abundance of the precursor ion post-fs-LID was 5.70E6, indicating a fragmentation efficiency of $70 \%$. This represents a substantial improvement compared with our initial report on the use of fs-LID for peptide dissociation [29], primarily due to the higher repetition rate of the laser employed in the current study. Abundant singly oxidized doubly charged and doubly oxidized triply charged radical product ions (i.e., $[\mathrm{M}+\mathrm{H}]^{2+\cdot}$ and $[\mathrm{M}+\mathrm{H}]^{3+\cdot \cdot)}$ ) were observed, as well as a wide variety of $a-, b-, c^{-}, x_{-}^{-}, y^{-}$, and $z$-type sequence ions from which complete sequence coverage could be achieved. The presence of the $x_{8}$ and $x_{9}$ product ions may be used to unambiguously localize the phosphate group to the threonine residue. An abundant ' $Y$ ' ion [35], which has two fewer hydrogens than a conventional $y$-type ion, was also observed in the fs-LID-MS/MS spectrum (see the ion labeled $Y_{6}$ in Figure 1a). These ions, which are commonly observed under high-energy CID-MS/MS conditions, may arise through a 'shattering' mechanism by which the system undergoes prompt dissociation at the site of energy input owing to the instantaneous uptake of a large excess of energy [36]. It is interesting to consider that ' $Y$ ' ions are seen to occur N-terminal to proline in three of the four instances for which we have performed fs-LID MS/MS on proline containing peptides. Such specificity has been previously documented as occurring during high-energy CID-MS/MS [35]. No- 
(a)
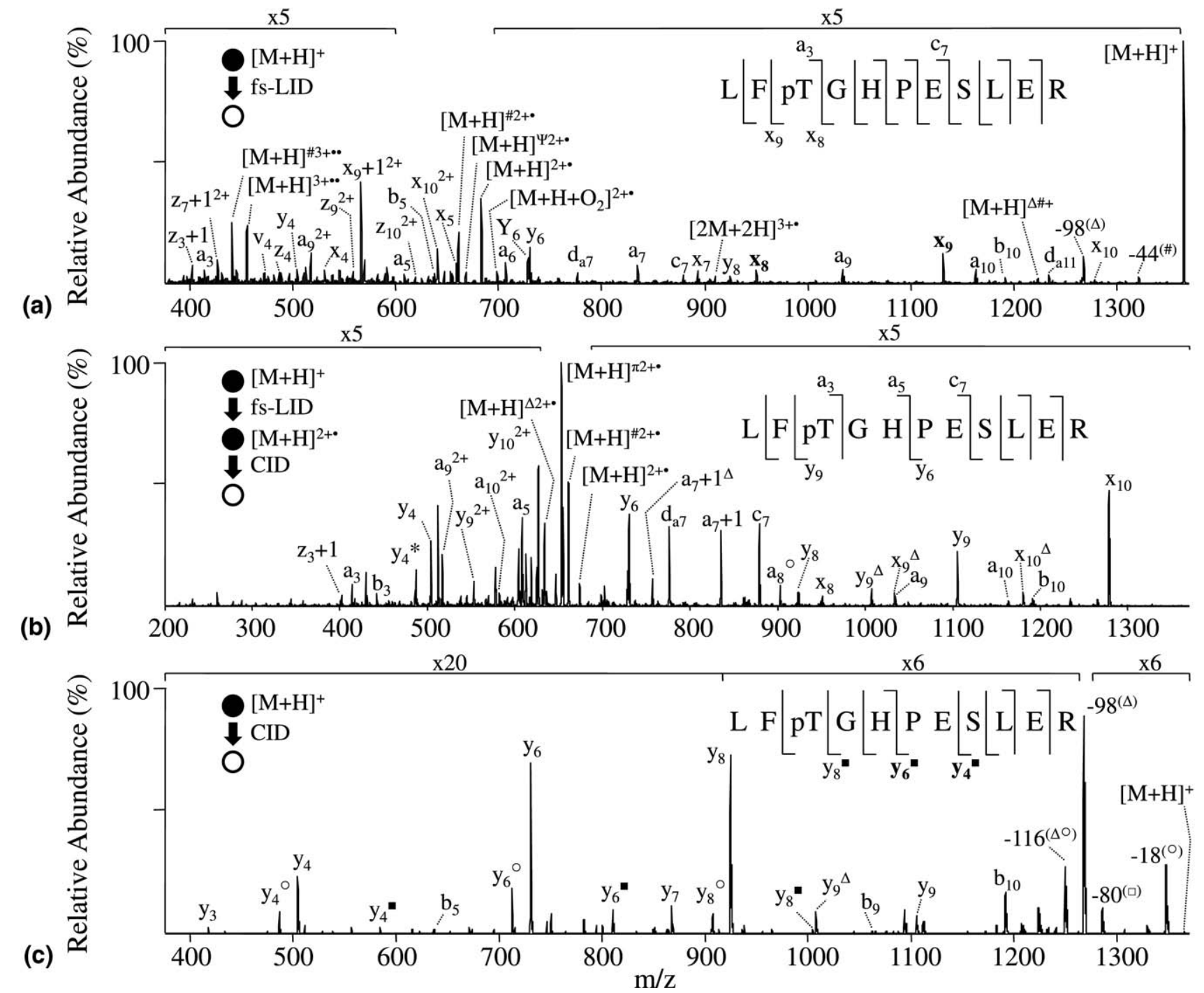

Figure 1. 3-D quadrupole ion trap mass spectra of the singly-phosphorylated peptide LFpTGHPESLER. (a) fs-LID-MS/MS of $[\mathrm{M}+\mathrm{H}]^{+},(\mathbf{b}) \mathrm{CID}^{-M S^{3}}$ of the $[\mathrm{M}+\mathrm{H}]^{2+}$ photo-oxidized product ion from (a), and (c) CID-MS/MS of $[\mathrm{M}+\mathrm{H}]^{+}$. Sequence ions that are most informative for localization of the phosphorylation site are labeled on the sequence insets. $*=$ loss of $17 \mathrm{Da} ; \mathrm{O}=1$ loss of $18 \mathrm{Da} ; \Psi=$ loss of $30 \mathrm{Da}$; $\#=$ loss of $44 \mathrm{Da} ; \pi=$ loss of $58 \mathrm{Da} ; \square=$ loss of $80 \mathrm{Da} ; \Delta=$ loss of 98 $\mathrm{Da} ; \boldsymbol{\square}=$ gain of $80 \mathrm{Da}$.

table too, ' $\mathrm{Y}$ ' ions have also been observed by $157 \mathrm{~nm}$ UVPD-MS/MS [37]. Other high-energy products observed by fs-LID-MS/MS analysis of the singlyprotonated LFpTGHPESLER peptide (again with similarity to UVPD-MS/MS) include satellite $d$ and $v$ ions.

In addition to the formation of sequence ions, the loss of small neutrals of $30 \mathrm{Da}\left(\mathrm{CH}_{2} \mathrm{O}\right.$, labeled with a $\left.\Psi\right)$ and $44 \mathrm{Da}\left(\mathrm{CO}_{2}\right.$ or $\mathrm{HNCHNH}_{2}$, labeled with a \#) were observed from the radical $[\mathrm{M}+\mathrm{H}]^{2+\cdot}$ and $[\mathrm{M}+\mathrm{H}]^{3+.}$ product ions formed by fs-LID of the singly-protonated LFPTGHPESLER peptide (Figure 1a), originating via side-chain fragmentation reactions and/or losses from the C-terminus. Similar losses have previously been reported by high-energy CID, ECD, ETD, EID, MAD, and UVPD [38, 33, 35, 39-41]. Finally, a low abundance product ion corresponding to the loss of $98 \mathrm{Da}$
$\left(-\mathrm{H}_{3} \mathrm{PO}_{4} \sim 2 \%\right.$ relative abundance) was observed from the precursor ion. In addition to tunneling ionization by which photo-ionization occurs, it is reasonable to assume that some amount of 'slow heating' IRMPDderived fragment ion production could occur during fs-LID, owing largely to the high photon flux and the long irradiation time $(100 \mathrm{~ms})$ currently used to achieve an appreciable $(>50 \%)$ precursor dissociation efficiency. The possibility of IRMPD processes is supported by the fact that such thermodynamically-derived products were observed despite 'clean' isolation spectra, i.e., the observed products were not formed by collisional activation during the precursor ion isolation step. Note however, that in contrast to UVPD in ion trap mass spectrometers [28], no neutral losses of the phosphate group were observed from the various product ions 


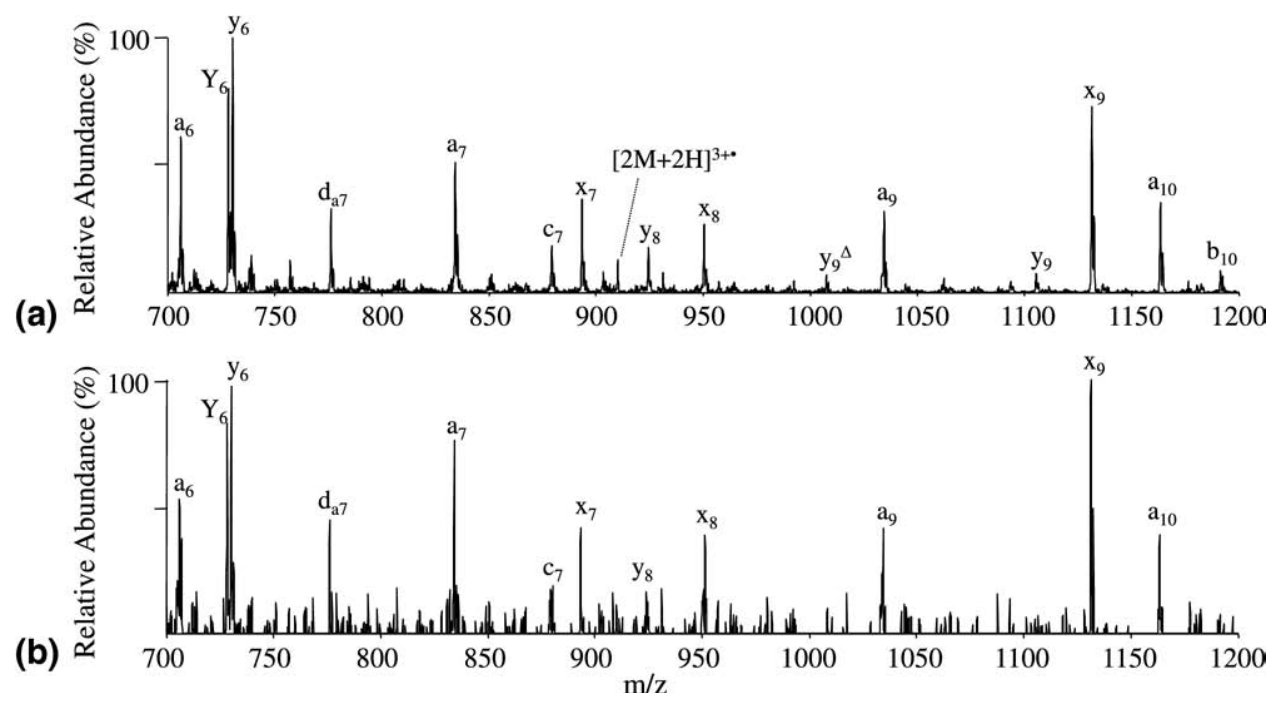

Figure 2. Expanded view of the fs-LID-MS/MS spectra acquired for the singly-phosphorylated peptide LFPTGHPESLER. Shown are the signal-averaged mass spectra of the $\mathrm{m} / \mathrm{z}$ range $700-1200$ for acquisition periods of (a) $3 \mathrm{~min}$, and (b) $10 \mathrm{~s}$.

formed via radical directed fragmentation pathways (i.e., $a, c, x$, and $z$ ions) for this peptide upon fs-LID.

Similar to that previously reported [29], CID-MS ${ }^{3}$ of the fs-LID-generated $[\mathrm{M}+\mathrm{H}]^{2+\cdot}$ doubly charged photo-oxidized product ion of the singly-protonated LFPTGHPESLER peptide reveals that many of the sequence product ions observed in the fs-LID-MS/MS spectrum originate via sequential ionization/dissociation processes (i.e., initial photo-ionization of the even electron $[\mathrm{M}+\mathrm{H}]^{+}$precursor ion followed by backbone dissociation of the resultant $[\mathrm{M}+\mathrm{H}]^{2+\cdot}$ ion) (Figure $1 \mathrm{~b}$ ). However, several of the product ions formed by fs-LIDMS/MS and CID-MS ${ }^{3}$ of the photo-oxidized fs-LID product are unique to one of the two spectra (e.g., the $x_{9}$ and $a_{7}$ products ion in Figure $1 \mathrm{a}$ and the $\mathrm{y}_{9}$ and $\mathrm{a}_{7}+1$ products in Figure 1b). Thus, while not performed here due to limitations of the data acquisition software, simultaneous activation of the oxidized radical product ion(s) during fs-LID could be used to not only increase the sensitivity of the analysis, but also to provide a source of additional complementary product ions for structural assignment.

The overall spectral quality and structural information contained within the fs-LID-MS/MS and MS $^{3}$ spectra in Figure 1a and $b$, respectively, compare very favorably with the CID-MS/MS product ion spectrum of the same peptide, shown in Figure 1c. This spectrum is characterized by a dominant loss of $98 \mathrm{Da}\left(\mathrm{H}_{3} \mathrm{PO}_{4}\right.$ or $\mathrm{HPO}_{3}+\mathrm{H}_{2} \mathrm{O}$, labeled with a superscript ' $\Delta$ ') from the precursor ion, losses of $98 \mathrm{Da}$ or $18 \mathrm{Da}\left(\mathrm{H}_{2} \mathrm{O}\right.$, labeled with a superscript ' $\mathrm{o}$ ') from the product ions [12], somewhat more limited sequence coverage, and the presence of $\mathrm{y}_{4}{ }^{*}, \mathrm{y}_{6}{ }^{*}$ (observed at a relative abundance of $\sim 15 \%$ that of the unmodified $\mathrm{y}_{6}$ ion) and $\mathrm{y}_{8} \cdot$ ions indicative of the phosphate group rearrangement reaction [12], all of which limit the ability to unambiguously assign the correct site of phosphorylation.
The fs-LID-MS/MS spectrum shown in Figure 1a is the average of individual spectra acquired for a period of $3 \mathrm{~min}$, to allow a detailed evaluation of the types of product ions that are formed, and the structural information that may be obtained, by using this technique. However, it is important to recognize that such long acquisition periods are not necessary, and that the majority of the informative product ions required for characterization of the peptide can be readily observed using acquisition periods of only a few seconds. For example, shown in Figure 2a is an expanded region $(\mathrm{m} / \mathrm{z}$ $700-1200$ ) of the product ion spectrum from Figure 1a ( 3 min acquisition), while Figure $2 b$ shows the same region of $m / z$ for a fs-LID-MS/MS spectrum acquired on the same peptide, for only $10 \mathrm{~s}$. Clearly, despite the expected decrease in signal-to-noise associated with the shorter acquisition period, the ability to identify and distinguish the majority of the 'real' product ions from noise is not compromised.

Another example of the potential of fs-LID-MS/MS for enhanced phosphopeptide characterization, and a demonstration of fs-LID's preferential utility for the dissociation of singly protonated precursor ions, is shown in Figure 3 for the singly (Figure 3a) and doubly (Figure $3 \mathrm{~b}$ ) protonated precursor ions of the doubly phosphorylated peptide HEVSASpTQpSTPASSR. This sequence is representative of a relatively difficult phosphopeptide to characterize with respect to localization of the phosphate groups, as there are two phosphorylation sites, but a total of seven potential phosphorylation sites. In addition, the two phosphorylated residues lie directly adjacent to non-phosphorylated serine or threonine residues, thereby requiring cleavage between each of these residues for unambiguous phosphorylation site assignment. Similar to that shown in Figure 1, extensive sequence ion fragmentation was observed upon fs-LID of the singly protonated precursor (Figure 
(a)

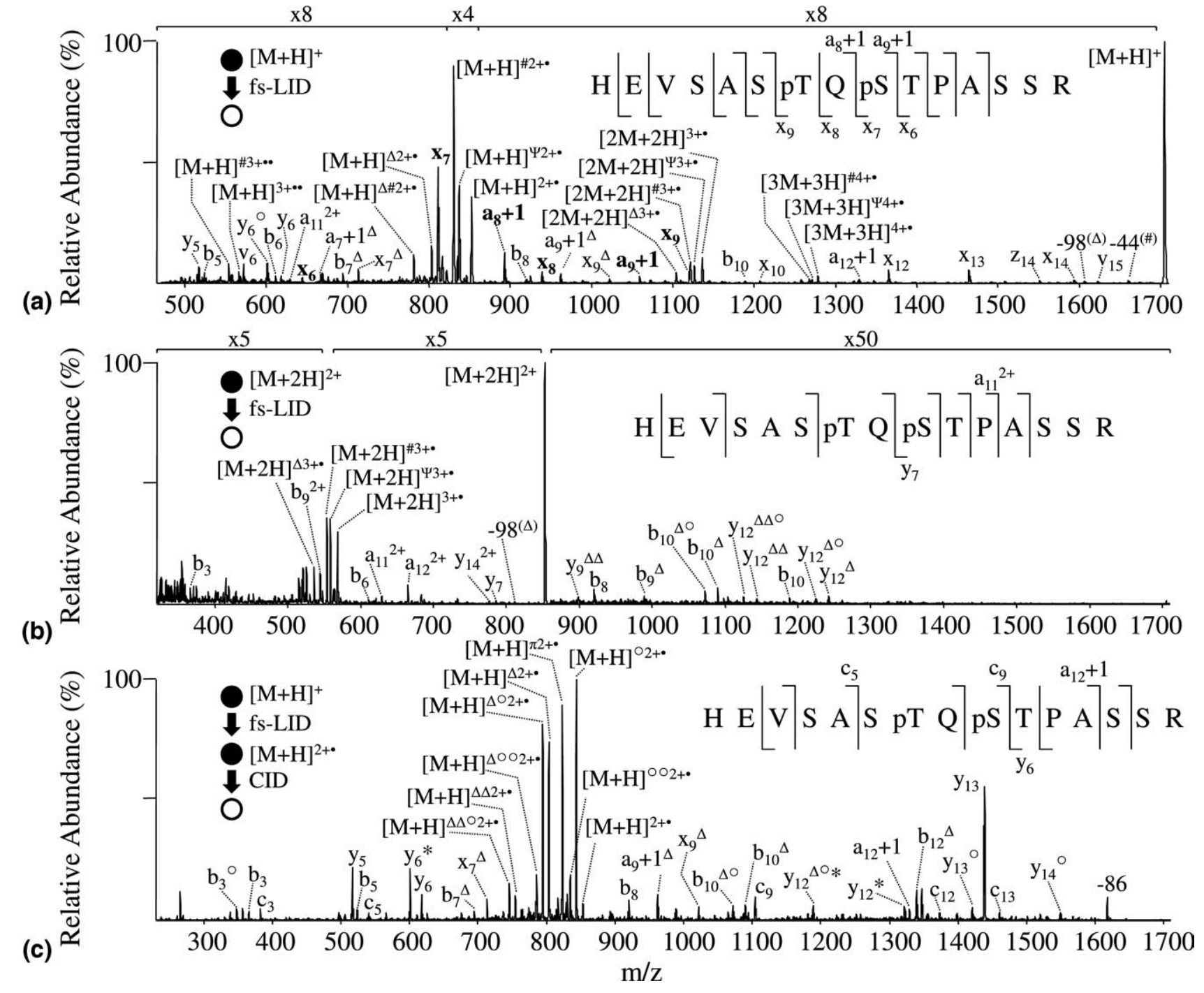

10 $\mathrm{x} 4$ $\mathrm{x} 8$

Figure 3. 3-D quadrupole ion trap mass spectra of the doubly-phosphorylated peptide HEVSASpTQpSTPASSR. (a) fs-LID-MS/MS of $[\mathrm{M}+\mathrm{H}]^{+}$, (b) fs-LID-MS/MS of $[\mathrm{M}+2 \mathrm{H}]^{2+}$, and (c) $\mathrm{CID}^{-\mathrm{MS}^{3}}$ of the $[\mathrm{M}+\mathrm{H}]^{2+\cdot}$ photo-oxidized product ion from panel A. Sequence ions which are most informative for localization of the phosphorylation sites are labeled on the sequence insets. ${ }^{*}=$ loss of $17 \mathrm{Da} ; \bigcirc=$ loss of $18 \mathrm{Da} ; \Psi=$ loss of $30 \mathrm{Da} ; \#=$ loss of $44 \mathrm{Da} ; \pi=$ loss of $58 \mathrm{Da} ; \Delta=$ loss of $98 \mathrm{Da}$.

3a), from which the two sites of phosphorylation could be readily assigned (via the $x_{6}, x_{7}, x_{8}, x_{9}, a_{8}+1$, and $\mathrm{a}_{9}+1$ product ions). With the exception of the $\mathrm{a}_{9}+1^{\Delta}$, $\mathrm{x}_{7}{ }^{\Delta}$, and $\mathrm{x}_{9}{ }^{\Delta}$ product ions (where the superscript ${ }^{\Delta}$ corresponds to the loss of $98 \mathrm{Da}, \mathrm{H}_{3} \mathrm{PO}_{4}$ ), all of the products formed via radical directed fragmentation pathways were observed to retain the phosphate moiety. As mentioned in the introduction, the observation of $a_{n}+1^{\Delta}$ ions have recently been reported using 193 nm UVPD in a tandem time-of-flight (TOF-TOF) instrument, and can be used to provide characteristic information regarding the location of the phosphate group within the peptide sequence [27]. The formation of $x_{n}{ }^{\Delta}$ ions identified in this study have not previously been reported. Interestingly, previously unreported $a_{n}+1^{\Delta}$ product ions were also observed in the ETD-MS/MS spectrum from the triply protonated precursor ions of the HEVSASpTQpSTPASSR and LFpTGHPESLER peptides (Supplemental Figures S1A and S1B, respectively, which can be found in the electronic version of this article). Elucidating the mechanism(s) responsible for the formation of these ions by fs-LID and ETD, and evaluation of their diagnostic potential for aiding phosphorylation site assignment, is the subject of separate investigation in our laboratory, the results of which will be reported elsewhere.

Figure $3 \mathrm{c}$ shows the CID-MS ${ }^{3}$ spectrum of the doubly charged radical product ion $\left([\mathrm{M}+\mathrm{H}]^{2+}\right.$ ) from Figure 3a. Although the losses of small neutral species from the precursor ion dominated the spectrum, some additional complementary product ions were observed compared with those seen in the fs-LID-MS/MS spectrum, similar to the results described above for the LFPTGHPESLER peptide in Figure 1. 
To again highlight the detailed structural information that can be obtained by fs-LID, even with short acquisition periods, an expanded $\mathrm{m} / \mathrm{z}$ region of the signal-averaged fs-LID-MS/MS spectrum from Figure 3a (acquisition period of $3 \mathrm{~min}$ ) is shown in Supplemental Figure S2A, while the signal-averaged spectrum for an acquisition period of $10 \mathrm{~s}$ is shown in Supplemental Figure S2B. Consistent with that discussed in Figure 2, despite the decrease in signal-tonoise, observation of the majority of the ions required to characterize the peptide sequence and sites of phosphorylation are not compromised by using the shorter acquisition period.

In contrast to the results obtained for the singly protonated precursor ion, fs-LID of the doubly protonated precursor of the HEVSASpTQpSTPASSR peptide (Figure $3 b$ ) resulted in inefficient dissociation and radical initiated product ion formation, limiting characterization of both the peptide sequence and sites of phosphorylation. Indeed, comparison of the fs-LID-MS/MS data in Figure $3 \mathrm{~b}$ with the isolation spectra (data not shown) suggested that the majority of the observed product ions arise from thermodynamically-derived fragmentation pathways (e.g., phosphate moiety losses and $\mathrm{b} / \mathrm{y}$ ion production) due to either off-resonant excitation during isolation or the occurrence of IRMPD over the course of the relatively long activation period (100 ms). Similarly, limited fs-LID-MS/MS dissociation efficiencies were observed for all the other multiplyprotonated phosphopeptides studied to date (data not shown). As stated in the Introduction section, and similar to that previously reported by EID [31] and $\operatorname{MAD}[32,33]$, this phenomenon is attributed to the higher ionization potential of multiply protonated peptide precursor ions compared with singly protonated precursors [31]. Thus, as our results indicate that photoelectron ionization plays a key initiating role in the fs-LID mechanism, ionization potential is expected to be the major limiting factor to the technique's efficiency.

Supplemental Figure S3 shows the CID-MS/MS product ion spectra for the singly, doubly, and triply protonated precursor ions for the HEVSASpTQPSTPASSR peptide. Consistent with our previous reports [11, 12], dominant losses of the phosphate moieties [e.g., $\left.-\mathrm{HPO}_{3}(\square, 80 \mathrm{Da}),-\mathrm{H}_{3} \mathrm{PO}_{4}(\Delta, 98 \mathrm{Da})\right]$ as well as sequential losses of phosphate groups and water [e.g., $\Delta \Delta$ and $\Delta \bigcirc$ ] from both the precursor ion and the product ions are observed under non-mobile and partially-mobile protonation conditions (see Figures S3A and S3B for the $[\mathrm{M}+\mathrm{H}]^{+}$and $[\mathrm{M}+2 \mathrm{H}]^{2+}$ ions, respectively) [11, 12]. Furthermore, product ions formed via phosphate group rearrangement reactions [e.g., $\mathrm{y}_{5}{ }^{*}$ and $\mathrm{y}_{6}{ }^{*}$ in Figure S3A and $\mathrm{y}_{6}{ }^{*}$ in Figure S3B] were also observed as relatively abundant species ( $\sim 10 \%-20 \%$ compared with their corresponding unmodified y-ions) in these spectra. Notably, however, neutral losses from the triply-protonated precursor ion (Figure S3C), classified as having 'mobile protons', were significantly reduced, and product ions formed via rearrangement reactions were not observed. Thus, as proposed in the introduction section, fs-LID can be employed as a complementary methodology for the characterization of singly protonated phosphopeptide precursor ions, i.e., those which are currently least reliably analyzed by CID-MS/MS, or are not amenable to ETD-MS/MS.

In the course of investigating the fs-LID-MS/MS of singly-protonated phosphopeptides, several anomalous ions were observed. Inspection of the isotopic envelopes for these ions determined that these species were formed via photo-ionization of multiply protonated phosphopeptide dimer and trimer clusters, that were co-isolated with the singly protonated monomeric precursor ion species, and had the form $[\mathrm{nM}+\mathrm{nH}]^{(n+1)+}$. (e.g., see the peaks labeled $[2 \mathrm{M}+2 \mathrm{H}]^{3+\cdot}$ in Figures 1a and $3 \mathrm{a}$, and $[3 \mathrm{M}+3 \mathrm{H}]^{4+\cdot}$ in Figure $3 \mathrm{a}$ ). Other ions associated with the clusters were observed to correspond to the losses of small neutral species, with similar abundances to the monomeric photo-oxidized ions. The origins of the clusters were attributed to the solutionphase formation of strong intermolecular noncovalent bonds involving the phosphate moieties and protonated basic amino acid side chains, as previously reported [22]. Attempts to reduce the abundance of the cluster ions in this peptide by sample dilution to $2 \mu \mathrm{M}$ did not prevent their formation. Though mild in-source collisional heating was observed to markedly reduce the clusters, in-source CID was not utilized for the data reported herein, as such conditions could promote undesirable phosphate rearrangement. Since co-isolation of these multimeric clusters occurs along with the intended monomeric precursor species, the resultant products must be taken into consideration to avoid erroneous product ion assignments.

Additional examples demonstrating the applicability of fs-LID-MS/MS for the characterization of singly protonated phosphopeptide ions are shown in Figure 4 (HYGIpTSPISLAAPK), and in Supplemental Figure S4 (LNQSpSPDNVTDTK), Figure S5 (EDpSGTFSLGK), and Figure S6 (SLSSPpTDNLELSLR). Notably, ESI-MS analysis of each of these peptides either did not yield an $[\mathrm{M}+3 \mathrm{H}]^{3+}$ precursor ion, or the $[\mathrm{M}+3 \mathrm{H}]^{3+}$ precursor ion was only observed at very low abundance, thereby precluding the ability to acquire ETD-MS/MS spectra. Furthermore, in some cases, ETD-MS/MS of the abundant $[\mathrm{M}+2 \mathrm{H}]^{2+}$ precursor ions from these peptides resulted mainly in the formation of the charge reduced $[\mathrm{M}+2 \mathrm{H}]^{+\cdot}$ product and associated neutral losses, such that the most abundant sequence ions were only observed at very low relative abundance (e.g., $<1 \%$ ), even with the use of supplemental activation (data not shown). Thus, the information provided by fs-LIDMS/MS of the singly protonated precursor ions can be critical to achieve phosphopeptide identification and characterization. 
(a)

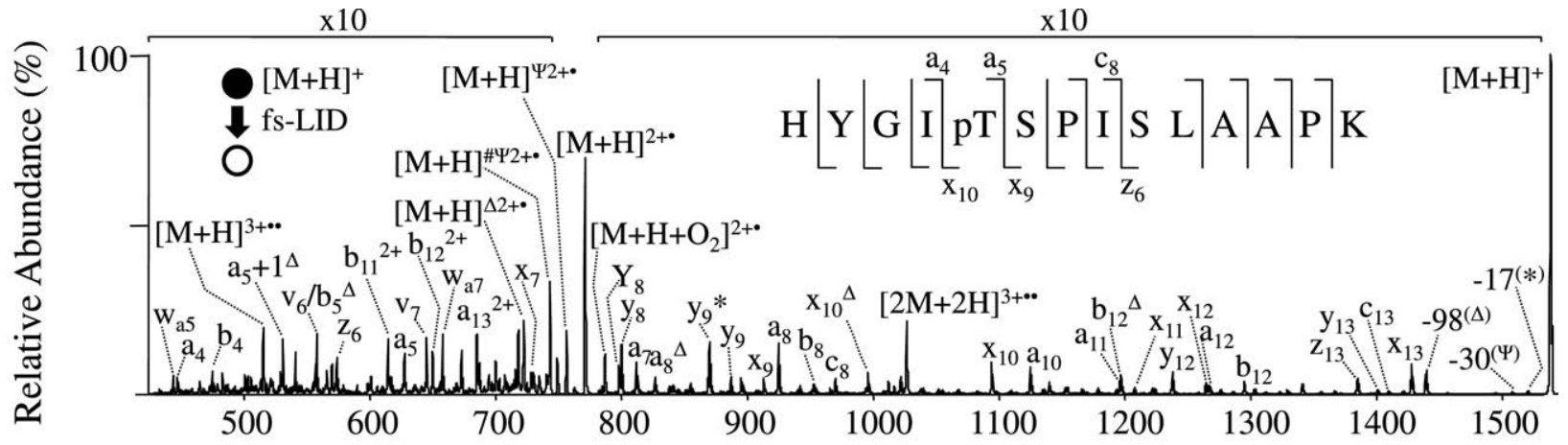

(b)

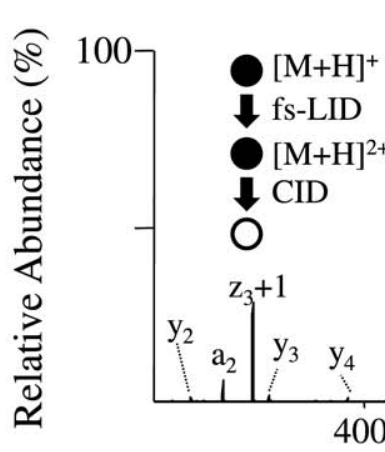

$$
[\mathrm{M}+\mathrm{H}]^{\mathrm{O} 2+\bullet}
$$

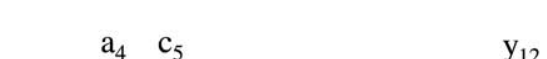

$\mathrm{y}_{12}$

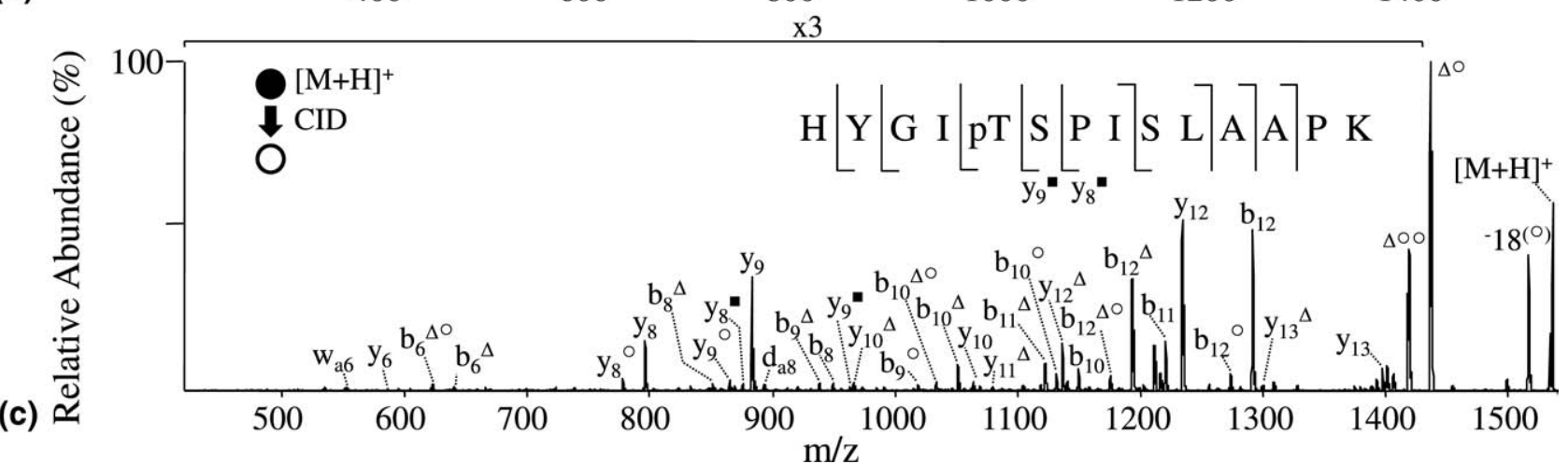

Figure 4. 3-D quadrupole ion trap mass spectra of the singly-phosphorylated peptide HYGIpTSPISLAAPK. (a) fs-LID-MS/MS of $[\mathrm{M}+\mathrm{H}]^{+}$, (b) CID-MS ${ }^{3}$ of the $[\mathrm{M}+\mathrm{H}]^{2+}$ photo-oxidized product ion from in (a), and (c) CID-MS/MS of $[\mathrm{M}+\mathrm{H}]^{+}$. Sequence ions which are most informative for localization of the phosphorylation site are labeled on the sequence insets. $*=$ loss of $17 \mathrm{Da} ; \bigcirc=$ loss of $18 \mathrm{Da} ; \Psi=$ loss of $30 \mathrm{Da} ; \#=$ loss of $44 \mathrm{Da} ; \xi=$ loss of $56 \mathrm{Da} ; \Delta=$ loss of $98 \mathrm{Da} ; \mathbf{\square}=$ gain of $80 \mathrm{Da}$.

\section{Conclusions}

In this study, fs-LID-MS/MS was characterized for its applicability to the analysis of singly protonated phosphopeptides. The technique is capable of providing comprehensive peptide sequence information via the production of radical-initiated sequence ions (i.e., $a, c, x$, and $z$ ions) without neutral losses or rearrangement of the phosphate groups, as well as potentially diagnostic $a_{n}+1-98$ and $x_{n}-98$ product ions, thereby allowing unambiguous characterization of the site(s) of phosphorylation. Signal averaged spectra with sufficient dissociation efficiencies and signal to noise ratios for product ion assignment can be obtained using an acquisition period of only $10 \mathrm{~s}$. The overall capacity of the fs-LID-, CID-, and ETD-MS/MS techniques for unambiguous phosphopeptide sequence assessment has been examined for a variety of parameters, including precursor charge state, proton mobility, and the susceptibility to phosphate group loss or rearrangement. CID and ETD are most appropriately applied to the analysis of multiply protonated phosphopeptides, while fs-LID is best applied to singly-protonated precursor ions. Future efforts directed toward increasing the efficiency of the dissociation reaction (e.g., by further optimization of the overlap of the trapped ion population with the laser pulses, and via the use of supplemental activation of the photo-ionized intermediates), as well as coupling of the fs-LID-MS/MS technique with a MALDI-equipped linear ion trap mass spectrometer (for increased sensitivity and the efficient formation of singly protonated pep- 
tides), is expected to facilitate the broader applicability of fs-LID for phosphoprotein characterization.

\section{Acknowledgments}

The authors acknowledge support for this work by the National Science Foundation grant CHE-0547940, and from grant 156 of the 21st Century Jobs Trust Fund of the SEIC Board from the State of Michigan. C.L.K. thanks the National Science Foundation for graduate student fellowship funding. K.A.S. thanks Michigan State University for funding through an undergraduate Professorial Assistantship. The authors thank Dr. Amanda M. Palumbo for insightful discussions relating to the gas-phase ion chemistry of protonated phosphopeptides.

\section{Appendix A Supplementary Material}

Supplementary material associated with this article may be found in the online version at doi:10.1016/ j.jasms.2010.08.016.

\section{References}

1. Cohen, P. The Regulation of Protein Function by Multisite Phosphorylation-a 25 Year Update. Trends Biochem. Sci. 2000, 25, 596-601.

2. Bode, A. M.; Dong, Z. Post-Translational Modification of p53 in Tumorigenesis. Nat. Rev. Cancer 2004, 4, 793-805.

3. Mazanetz, M. P.; Fischer, P. M. Untangling $\tau$ Hyperphosphorylation in Drug Design for Neurodegenerative Diseases. Nat. Rev. Drug Discov. 2007, 6, 464-479.

4. Gong, C.-X., Liu, F.; Grundke-Iqbal, I.; Iqbal, K. Dysregulation of Protein Phosphorylation/Dephosphorylation in Alzheimer's Disease: A Therapeutic Target. J. Biomed. Biotechnol. 2006, 2006, 1-11.

5. Paradela, A.; Albar, J. P. Advances in the Analysis of Protein Phosphorylation. J. Prot. Res. 2008, 7, 1809-1818.

6. Boersema, P. J.; Mohammed, S.; Heck, A. J. R. Phosphopeptide Fragmentation and Analysis by Mass Spectrometry. J. Mass Spectrom. 2009, 44, 861-878.

7. Palumbo, A. M.; Smith, S. A.; Kalcic, C.L.; Dantus, M.; Stemmer, P. M.; Reid, G. E. Tandem Mass Spectrometry Strategies for Phosphoproteome Analysis. Mass Spectrom. Rev. 2010, in Press.

8. Dunn, J. D.; Reid, G. E.; Bruening, M. L. Enrichment of Phosphopeptides Prior to Analysis by Mass Spectrometry. Mass Spectrom. Rev. 2010, 29, 29-54.

9. Olsen, J. V.; Blagoev, B.; Gnad, F.; Macek, B.; Kumar, C.; Mortensen, P.; Mann, M. Global, In Vivo and Site-Specific Phosphorylation Dynamics in Signaling Networks. Cell 2006, 127, 635-648.

10. Beausoleil, S. A.; Villén, J.; Gerber, S. A.; Rush, J.; Gygi, S. P. A Probability-Based Approach for High-Throughput Protein Phosphorylation Analysis and Site Localization. Nat. Biotechnol. 2006, 24, 12851292.

11. Palumbo, A. M.; Tepe, J. J.; Reid, G. E. Mechanistic Insights into the Multistage Gas-Phase Fragmentation Behavior of Phosphoserine- and Phosphothreonine-containing Peptides. J. Protein Res. 2008, 7, 771-779.

12. Palumbo, A. M.; Reid, G. E. Evaluation of Gas-Phase Rearrangement and Competing Fragmentation Reactions on Protein Phosphorylation Site Assignment using CID-MS/MS and $\mathrm{MS}^{3}$. Anal. Chem. 2008, 80, 9735-9747.

13. Sweet, S. M. M.; Creese, A. J.; Cooper, H. J. Strategy for the Identification of Sites of Phosphorylation in Proteins: Neutral Loss Triggered Electron Capture Dissociation. Anal. Chem. 2006, 78, 7563-7569.

14. Aguiar, M.; Haas, W.; Beausoleil, S. A.; Rush, J.; Gygi, S. P. Gas-Phase Rearrangements Do Not Affect Site Localization Reliability in Phosphoproteomics Data Sets. J. Proteome Res. 2010, 9, 3103-3107.

15. Mischerikow, N.; Altelaar, A. F. M.; Navarro, J. D.; Mohammed, S.; Heck, A. Comparative Assessment of Site Assignments in CID and ETD Spectra of Phosphopeptides Discloses Limited Relocation of Phosphate Groups. Mol. Cell. Proteom. 2010, in press; doi:10.1074/mcp. M900619-MCP200.
16. Sweet, S. M. M.; Bailey, C. M.; Cunningham, D. L.; Heath, J. K.; Cooper, H. J. Large Scale Localization of Protein Phosphorylation by Use of Electron Capture Dissociation Mass Spectrometry. Mol. Cell. Proteom. 2009, 8, 904-912.

17. Syka, J. E. P.; Coon, J. J.; Schroeder, M. J.; Shabanowitz, J.; Hunt, D. F. Peptide and Protein Sequence Analysis by Electron Transfer Dissociation Mass Spectrometry. Proc. Natl. Acad. Sci. U.S.A. 2004, 101, 95289533.

18. Molina, H.; Horn, D. M.; Tang, N.; Mathivanan, S.; Pandey, A. Global Proteomic Profiling of Phosphopeptides Using Electron Transfer Dissociation Tandem Mass Spectrometry. Proc. Natl. Acad. Sci. U.S.A. 2007, 104, 2199-2204

19. Swaney, D. L.; Wenger, C. D.; Thomson, J. A.; Coon, J. J. Human Embryonic Stem Cell Phosphoproteome Revealed by Electron Transfer Dissociation Tandem Mass Spectrometry. Proc. Natl. Acad. Sci. U.S.A. 2009, 106, 995-1000.

20. Chalkley, R. J.; Medzihradszky, K. F.; Lynn, A. J.; Baker, P. R.; Burlingame, A. L. Statistical Analysis of Peptide Electron Transfer Dissociation Fragmentation Mass Spectrometry. Anal. Chem. 2010, 82, 579-584.

21. McLuckey, S. A.; Huang, T. Y. Ion/Ion Reactions: New Chemistry for Analytical MS. Anal. Chem. 2009, 81, 8669-8676.

22. Jackson, S. N.; Wang, H. Y. J.; Yergey, A.; Woods, A. S. Phosphate Stabilization of Intermolecular Interactions. J. Proteome Res. 2006, 5, 122-126.

23. Swaney, D. L.; McAlister, G. C.; Wirtala, M.; Schwartz, J. C.; Syka, J. E. P.; Coon, J. J. Supplemental Activation Method for High-Efficiency Electron-Transfer Dissociation of Doubly Protonated Peptide Precursors. Anal. Chem. 2007, 79, 477-485.

24. Brodbelt, J. S.; Wilson, J. J.; Infrared Multiphoton Dissociation in Quadrupole Ion Traps. Mass Spectrom. Rev. 2009, 28, 390-424.

25. Reilly, J. P. Ultraviolet Photofragmentation of Biomolecular Ions. Mass Spectrom. Rev. 2009, 28, 425-447.

26. Ly, T.; Julian, R. R. Ultraviolet Photodissociation: Developments Towards Applications for Mass-Spectrometry-Based Proteomics. Angew. Chem. Int. Ed. 2009, 48, 7130-7137.

27. Shin, Y. S.; Moon, J. H.; Kim, M. S. Observation of Phosphorylation Site-Specific Dissociation of Singly Protonated Phosphopeptides.J. Am. Soc. Mass Spectrom. 2010, 21, 53-59.

28. Kim, T.-Y.; Reilly, J. P. Time-Resolved Observation of Product Ions Generated by $157 \mathrm{~nm}$ Photodissociation of Singly Protonated Phosphopeptides.J. Am. Soc. Mass Spectrom. 2009, 20, 2334-2341.

29. Kalcic, C. L.; Gunaratne, T.; Jones, A. D.; Dantus, M.; Reid, G. E. Femtosecond Laser-induced Ionization/Dissociation of Protonated Peptides. J. Am. Chem. Soc. 2009, 131, 940-942.

30. Zewail, A. H. Femtochemistry: Atomic-Scale Dynamics of the Chemical Bond Using Ultrafast Laser. Angew. Chem. Int. Ed. 2000, 39, 2586-2631.

31. Budnik, B. A.; Tsybin, Y. O.; Hakansson, P.; Zubarev, R. A. Ionization Energies of Multiply Protonated Polypeptides Obtained by Tandem Ionization in Fourier Transform Mass Spectrometers. J. Mass Spectrom. 2002, 37, 1141-1144.

32. Cook, S. L.; Collin, O. L.; Jackson, G. P. Metastable Atom-Activated Dissociation Mass Spectrometry: Leucine/Isoleucine Differentiation and Ring Cleavage of Proline Residues. J. Mass Spectrom. 2009, 44, 1211-1223.

33. Berkout, V. D.; Doroshenko, V. M. Fragmentation of Phosphorylated and Singly Charged Peptide Ions Via Interaction with Metastable Atoms. Int. J. Mass Spectrom. 2008, 278, 150-157.

34. Coello, Y.; Lozovoy, V. V.; Gunaratne, T. C.; Xu, B.; Borukhovich, I.; Tseng, C-h.; Weinacht, T.; Dantus, M. Interference Without an Interferometer: A Different Approach to Measuring, Compressing, and Shaping Ultrashort Laser Pulses.J. Opt. Soc. Am. B 2008, 25, A140-A150.

35. Medzihradszky, K. F.; Burlingame, A. L. The Advantages and Versatility of a High-Energy Collision-Induced Dissociation-Based Strategy for the Sequence and Structural Determination of Proteins. Methods 1994, 6, $284-303$.

36. Laskin, J.; Bailey, T. H.; Futrell, J. H. Shattering of Peptide Ions on Self-Assembled Monolayer Surfaces. J. Am. Chem. Soc. 2003, 125, 16251632.

37. Cui, W. D.; Thompson, M. S.; Reilly, J. P. Pathways of Peptide Ion Fragmentation Induced by Vacuum Ultraviolet Light. J. Am. Soc. Mass Spectrom. 2005, 16, 1384-1398.

38. Fung, Y. M. E.; Adams, C. M.; Zubarev, R. A. Electron Ionization Dissociation of Singly and Multiply Charged Peptides. J. Am. Chem. Soc. 2009, 131, 9977-9985.

39. Cooper, H. J.; Hudgins, R. R.; Hakansson, K.; Marshall, A. G. Characterization of Amino Acid Side Chain Losses in Electron Capture Dissociation.J. Am. Soc. Mass Spectrom. 2002, 13, 241-249.

40. Good, D. M.; Wenger, C. D.; McAlister, G. C.; Bai, D. L.; Hunt, D. F.; Coon, J. J. Post-Acquisition ETD Spectral Processing for Increased Peptide Identifications. J. Am .Soc. Mass Spectrom. 2009, 8, 1435-1440.

41. Kjeldsen, F.; Silivram, O. A.; Zubarev, R. A. Zwitterionic States in Gas-Phase Polypeptide Ions Revealed by 157-nm Ultra-Violet Photodissociation. Chem. Eur. J. 2006, 12, 7920-7928. 